\title{
VIRTUAL NETWORKING LABORATORY FOR EDUCATION IN COMPUTER SCIENCE
}

\author{
Jordan H. Kanev', \\ Stanimir M. Sadinov ${ }^{1}$ \\ ${ }^{1}$ Technical University of Gabrovo, \\ Gabrovo, Bulgaria
}

\begin{abstract}
:
Teaching distance learning or e-learning classes include online lectures, exams and laboratory exercises using real equipment and/or simulators. This paper proposes a way to create a virtual laboratory, which provides distant access to real and virtual equipment without the risk of losing access due to misconfiguration of devices.
\end{abstract}

Keywords:

Distance learning, Virtual Laboratory, Remote access.

\section{INTRODUCTION}

In the modern dynamic life people demand more flexible education that fits their needs and daily time schedule. In learning concepts, the role of e-learning platforms and virtual labs is growing rapidly. There are many sophisticated online learning systems offering learning content management and delivering teaching data. This is one of the building blocks for the e-learning and distance learning process. But for the most of the bachelor and master degree courses for engineers this is not enough. For example, nearly half of the education process for engineers in the scientific field "Communication and computer technics" at our university is being conducted in labs, doing experiments, demonstrating processes and working with equipment. Students are divided in groups of ten to fifteen people. If for example dynamic routing must be demonstrated, every student will need minimum three routers, which makes a total of 30 to 45 routers. In the year 2014, our colleague Delyan Genkov [1] proposed a way to create a virtual laboratory, which provides distant access to real equipment without the risk of losing access due to misconfiguration of devices. The main disadvantage of this system is that the whole group of students cannot use it simultaneously and they have to wait for their turn to work with the equipment. Another disadvantage is that there are three to four groups of students working on the same exercise and once the group has finished, the configurations have to be cleared and initial setups uploaded.

Another approach is presented by Valchanov and Yuseinov [4]. They are proposing a virtual Cisco laboratory with provided access to simulated, instead of real devices. In our opinion this way students will not have e-mail:

ykanev@tugab.bg
Correspondence:

Jordan H. Kaney 
access to real devices and they will get a wrong impression about the behaviour of devices. For example the router Cisco 2821 boots in two to three minutes. In some simulators [5-10] the device boots within seconds and when students try to work with real instead of simulated devices they think that the device has malfunctioned or is faulty, because it takes it too long to get operational.

Our goal is to provide students with enough networking devices, which contain a mix of real and simulated equipment, so they can fulfill laboratory exercises without the mentioned disadvantages. In addition, we try to equip every student with his own set of devices to work with, so there are no collusions between them.

\section{LABORATORY EQUIPMENT}

The laboratory base consists of ten workstations for students, one presenter personal computer equipped with a multimedia projector, communication rack with Cisco Networking Academy Program routers and switches and 16-port VLAN capable gigabit Ethernet switch for interconnections. Here are specifications of the equipment:

\section{Hardware}

- 1 x HPE ProLiant ML350 Gen9 Server

- 10x HP Com Pro 4300 Celeron 2,6Ghz/8GB/500GB

- $4 \mathrm{x}$ router Cisco 2821

- 1 x router Cisco 2511

- 2 x switch Cisco 2960

- 2 x switch Cisco 3560

- 1 x TP-Link Gigabit 16 port

Software and licenses

- 1 x Windows server 2012 Standard R2 64-bit

- $1 \mathrm{x}$ Windows 10 Pro 64-bit

- 1 x VMware ESXi 5.5.0 U2

- $10 \mathrm{x}$ Windows 7 Professional 64-bit

- 10 x Linux Debian 8 “jessie" 64-bit

- 1 x Linux Ubuntu Server 16.04.2 LTS 64-bit

All workstations are preinstalled with a dual boot option for Windows/Linux operating system. The teacher workstation is installed with Linux Ubuntu server operating system. The laboratory server is located inside the university server room in the same building, it holds a virtual platform VMware ESX, and runs two virtual machines with Windows server 2012 and Windows 10. The second virtual machine is used by teachers via RDP to present windows specific features, without the need to restart the presenting computer. This computer is used for the terminal server connection as well. Its serial port is connected to the console port of access server (cisco 2511) and from there it allows out-of-band access to all eight cisco devices via asynchronous serial interfaces to console ports of all cisco devices in the communication rack [1]. There is SSH daemon running on the teacher's computer, so this option is available from any device in the network. The access server has 16 ports so it can be used to manage more devices in the future. The block diagram of this scenario is presented in Fig. 1. This way the teacher can, not only troubleshoot device configurations, but also show students configurations on a white board with a projector.

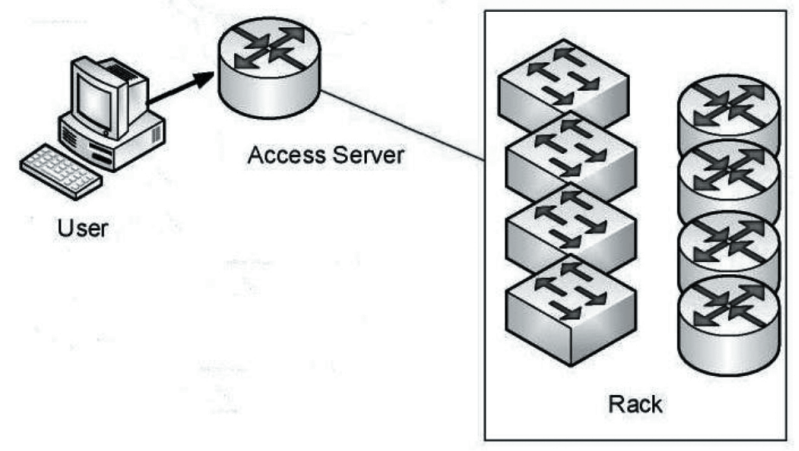

Fig. 1. Terminal server connection.

Even if a student loses connection to cisco devices, it is possible to access it via local network with SSH session to the access server. There is also a power management board connected to each device's power source. If a device needs to be restarted or reconfigured, this can be done via web interface [1].

\section{STUDENT WORKSTATIONS}

All workstations share the same parameters and hardware, so it is pretty easy to install all of them with a single image file. There is a dual boot option provided by grub boot loader and every student is provided with the choice of two operating systems when computer starts up.

There are two options:

- Windows 7 Professional

- Linux Debian

After loading the operating system, there is a different username and password for every discipline. For security reasons there is a different password for Windows and Linux user on the same discipline. There are specific tools and configurations for every user and operating system. 
All courses and topics performed in this lab are:

- Computer networks

- Internet technologies

- Internet services

- Databases

- Network security

- Surveillance systems

- Cisco Networking Academy courses

For every one of them there is a specific set of programs, tools and configurations. The major parameters, that must be unique for every workstation, are hostname and IP address. All other gradients should be the same for all computers.

Usually students do not have to configure or install anything on their workstations. Lower grade students even do not have administrative rights so they cannot install applications and perform system configurations. All of the exercises are virtualized, so there is no need to clear configurations after every student group.

Few years ago, we presented students with the opportunity to experience and work with all major virtualizing platforms $[14,15,16,17]$. The most suitable one turned out to be VirtualBox [15]. Even second grade students were able to import and start virtual machine in a few minutes. Combining real and virtualized devices $[6,9,10]$ we are able to demonstrate complex network scenarios, like the one demonstrated in Fig. 2.

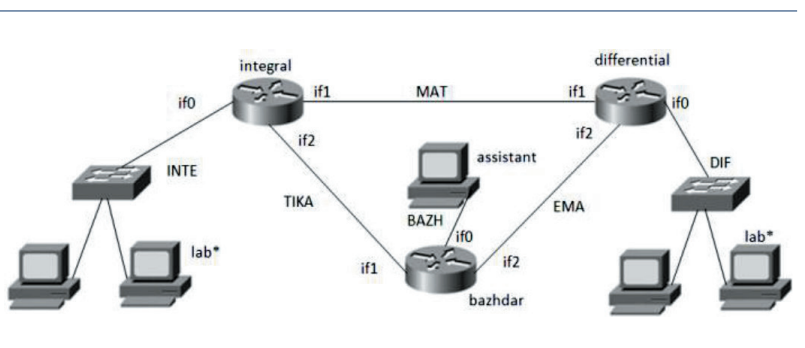

Fig. 2. Dynamic routing lab excersize.

When there are not enough real devices to fulfill lab exercise two simulators GNS3 and QEMU $[9,10]$ are put into action. They can simulate multiple devices simultaneously and allow a connection to the real local network for connection with real lab equipment. Fig. 3 shows a student workstation with GNS3 connected to the local network via SW1 switch.

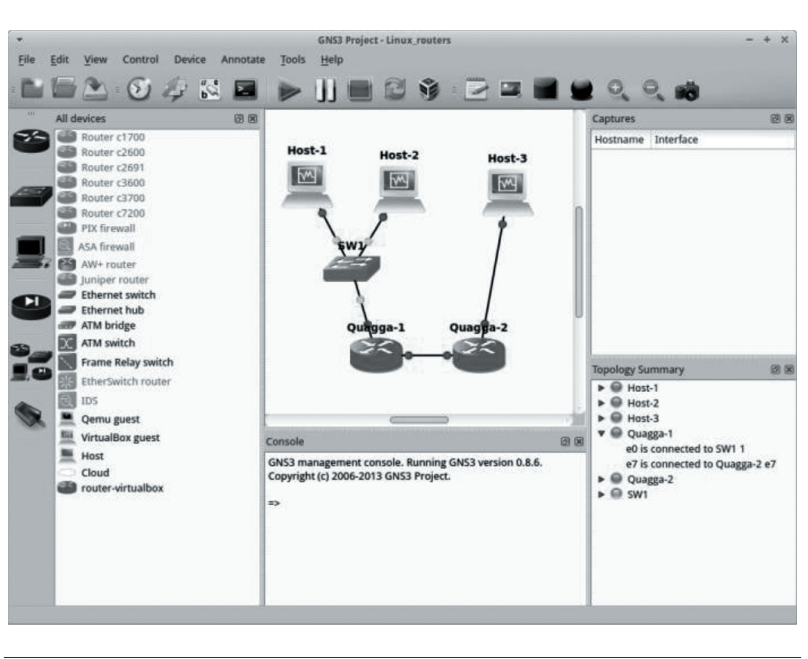

Fig. 3. GNS3 lab excersize simulation.

Another interesting fact is that students are making the same mistakes repeatedly. In order to assist teachers and to show all students common mistakes, we decide to install TightVNC server remote control software on all workstations. With one click of the mouse the teacher can open any student's desktop and show its content on the white board via a projector. VNC client application is shown in Fig. 4.

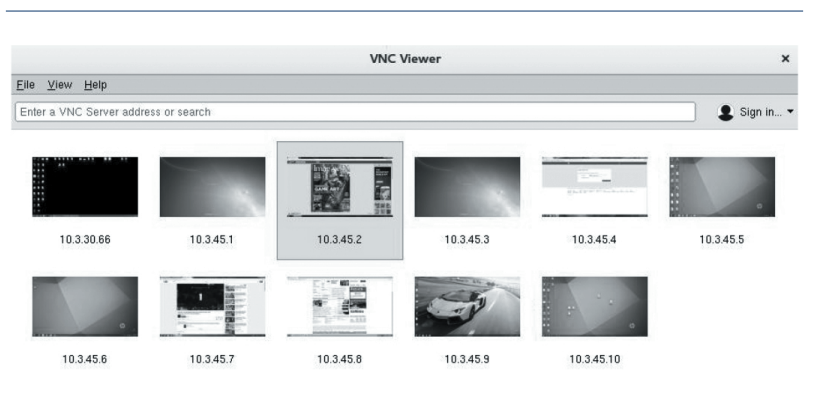

Fig. 4. VNC viewer connection screen.

In order for VNC to work properly there must be configured static IP addresses on all workstations or they should have reservations in DHCP server. Alternatively hostnames can be used in case there is a Windows active directory or DNS reverse lookup on the network.

\section{SERVER CONFIGURATION}

HP server is equipped with two hard drives, with one TB storage each. They are working together in array RAID 1 for fault tolerance. After HPE ILO configuration server is installed with virtualization hypervisor VMware ESXi 
[14]. There is $32 \mathrm{~GB}$ random access memory available for virtual machines, located on this server. If needed, RAM can be extended up to $3 \mathrm{~TB}$, based on 128 GB DDR4 LRDIMM. The system also has support for the new $25 \mathrm{GbE}$ Network Adapters and up to six hard drives, which gives possibilities for future upgrade and expansion. The server is managed with VMware vsphere client software (Fig. 5).

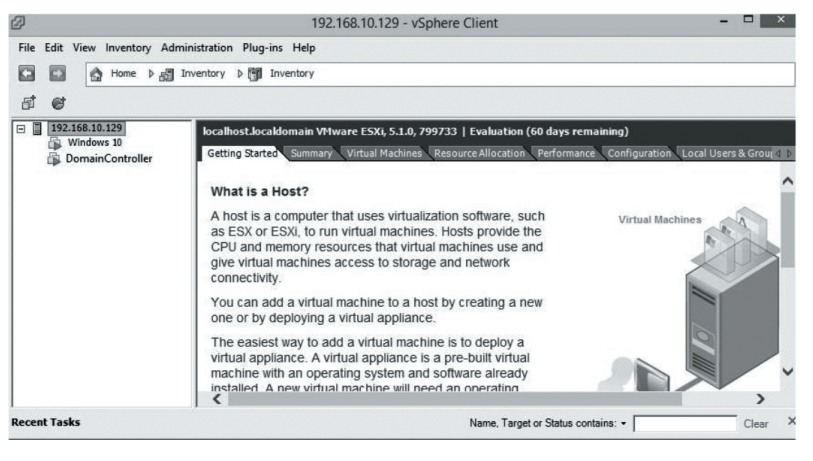

Fig. 5. VMware Vsphere Client 5.

Two virtual machines are installed on VMware platform. The first is based on Windows 10 64-bit operating system and its main goal is to be remotely accessed by teachers for presentations, installations and experiments. The second virtual machine is preinstalled with Windows server 2012 R2 64-bit operating system and its main role is to be a primary domain controller for "Computer systems and technologies" department in Technical university of Gabrovo. The website of department was relocated from the old server to this new virtual server at the end of last year. There is also Mysql database server, an internet forum and FTP server installed for administrative convenience.

Distance learning management system ILIAS is installed with the idea to upload all curriculum materials from CST department. All our students will then have full-time access to materials and lectures available online on the address http://kst.tugab.bg/ilias.

Future plans include collecting all important documents on this server and adding new services to teachers and students.

\section{CONCLUSION}

The paper proposes a way to organize a virtual laboratory with both real and virtual devices. It can be used to present lectures and laboratory exercises. It also provides the remote access to real equipment without the need of initial configurations of devices. This approach also protects users from losing the connection to the devices due to misconfiguration, which is a common issue with not very skilled students. It also provides a unique approach for presenting student desktops to auditory for troubleshooting complex networking issues. Laboratory exercises are very important for students studying computer networks and communications.

Direct elimination of a number of problems associated with maintenance, setup and system recovery after crash allows teaching efforts to be entirely focused on the learning process.

Some future enhancements are planned, including the creation of system backups and remote access to the resources of the laboratory, improved user control, accounting of the commands issued from the user and the possibility for automation of the assessment of configuration results.

\section{ACKNOWLEDGMENT}

The work presented in this paper is supported with assistance of two projects:

Project $1712 \mathrm{E}$ "Creating innovative information based educational training modules in communication techniques and technologies field"

Project 1709E "Development and implementation of innovative IT technologies in education"

Both projects are from University Center for Research and Technology at Technical University of Gabrovo.

\section{REFERENCES}

[1] D. Genkov, "Virtual Laboratory for Cisco Networking Academy Program", in proceedings of E-Learning'14 International Conference on E-learning, 12.09.2014, University of La Laguna, Spain, pp. 152 $-157$.

[2] S. Disston, Caicedo C. Approaches to the Design of Computer Networking Laboratories, ITERA 2010 conference, Nashville, TN, 2010.

[3] O. Tomov, "Virtual labs with remote access to a real hardware equipment in computer systems education", In proceedings of International Conference on Computer Systems and Technologies - CompSysTech'08, Gabrovo, pp V.17-1 - V.17-5.

[4] H. Valchanov, I. Yuseinov, A Virtualized Networking Laboratory, in proceedings of UNITECH 2012, Gabrovo, pp I473-I477.

[5] Boson NetSim, http://www.boson.com/netsim-cisco-network-simulator, date of use 20.02.2017 
[6] Cisco Packet Tracer, http://www.cisco.com/web/ learning/netacad/course_catalog/PacketTracer. html, date of use 20.02.2017

[7] The network simulator - NS2, http://www.isi.edu/ nsnam/ns/, date of use 1.02.2017

[8] Open Virtualization with KVM, http://www-03. $\mathrm{ibm} . c o m / s y s t e m s / p o w e r / s o f t w a r e /$ linux/powerkvm/index.html, date of use 13.02.2017

[9] QEMU the FAST! processor emulator, http://www. qemu-project.org/, date of use 10.02.2017

[10] GNS3 Documentation, https://docs.gns3.com/, date of use 25.02.2017

[11] TeamViewer, http://www.teamviewer.com, date of use 20.02.2017
[12] GoToMyPc, https://www.gotomypc.com, date of use 20.02.2017

[13] LogMeIn, https://secure.logmein.com/home, date of use 20.02.2017

[14] VMWare Hypervisor, http://www.vmware.com/ products/vsphere-hypervisor.html, date of use 20.02.2017

[15] VirtualBox Documentation, https://www.virtualbox.org/, date of use 20.02.2017

[16] Hyper-V - TechNet - Microsoft, https://technet. microsoft.com/en-us/library/mt169373(v=ws.11). aspx, date of use 20.02.2017

[17] Kernel Virtual Machine, https://www.linux-kvm. org/, date of use 20.02.2017 